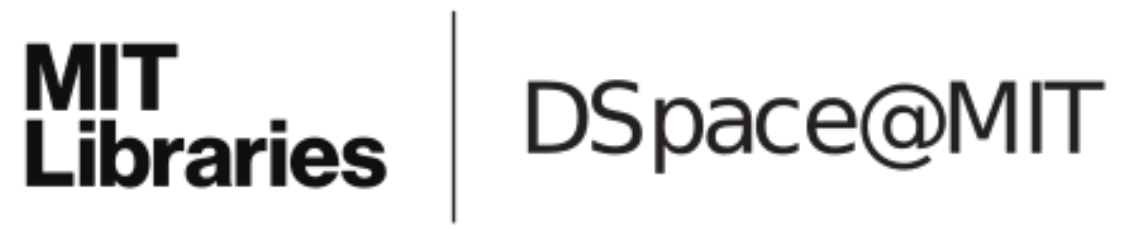

\author{
MIT Open Access Articles
}

\section{Multidisciplinary design optimization for hybrid electric vehicles: component sizing and multi-fidelity frontal crashworthiness}

The MIT Faculty has made this article openly available. Please share how this access benefits you. Your story matters.

As Published: https://doi.org/10.1007/s00158-020-02603-6

Publisher: Springer Berlin Heidelberg

Persistent URL: https://hdl.handle.net/1721.1/131926

Version: Author's final manuscript: final author's manuscript post peer review, without publisher's formatting or copy editing

Terms of Use: Article is made available in accordance with the publisher's policy and may be subject to US copyright law. Please refer to the publisher's site for terms of use. 


\section{Multidisciplinary Design Optimization for hybrid electric vehi- cles: component sizing and multi-fidelity frontal crashworthiness}

Cite this article as: P. G. Anselma, C. Boursier Niutta, L. Mainini and G. Belingardi, Multidisciplinary Design Optimization for hybrid electric vehicles: component sizing and multi-fidelity frontal crashworthiness, Structural and Multidisciplinary Optimization doi: $10.1007 / \mathrm{s} 00158-020-02603-6$

This Author Accepted Manuscript is a PDF file of a an unedited peer-reviewed manuscript that has been accepted for publication but has not been copyedited or corrected. The official version of record that is published in the journal is kept up to date and so may therefore differ from this version.

Terms of use and reuse: academic research for non-commercial purposes, see here for full terms. http://www.springer.com/gb/open-access/authors-rights/aam-terms-v1 


\title{
Multidisciplinary Design Optimization for hybrid electric vehicles: component sizing and multi-fidelity frontal crashworthiness
}

\author{
P. G. Anselma ${ }^{1} \&$ C. Boursier Niutta ${ }^{1} \&$ L. Mainini ${ }^{1,2} \&$ G. Belingardi ${ }^{1}$
}

\begin{abstract}
The electrification of road vehicle powertrains has recently gained growing interest worldwide as an effective solution to comply tightening regulations on $\mathrm{CO} 2$ emissions. In hybrid electric vehicles (HEVs), multiple power components, such as internal combustion engine, electric motor/generators and battery system, are included in the powertrain to improve vehicle performances, thus involving an increase of the powertrain envelope and of the overall vehicle mass. Larger vehicle mass affects the design of the structural framework, which expands its envelope to meet safety requirements. The expansions of both powertrain systems and structural framework are indeed constrained by the limited vehicle widthtrack, which demands for tradeoff studies at early vehicle design phases to achieve a feasible solution while optimizing performances related to different design disciplines. These particularly include proper sizing of power components to achieve optimal fuel economy capabilities and accurate design of structural components to satisfy crashworthiness criteria while minimizing the associated structural mass. In this framework, the achievement of a globally optimal solution requires a cooperative development process that addresses the design problem accounting for all these disciplinary contributions in an integrated manner. This paper proposes a Multidisciplinary Design Optimization (MDO) framework for the preliminary design of a power split HEV powertrain accounting for crashworthiness requirements. A multidisciplinary feasible optimization architecture is particularly illustrated that includes a sequential scheme to evaluate the disciplines and a direct search method as the global system optimizer. Different sizes are swept for the internal combustion engine, the electric motors and the planetary gear ratios. A multi-fidelity approach
\end{abstract}

1 Politecnico di Torino, Turin, Italy, [Pier.Anselma, Carlo.Boursier, Giovanni.Belingardi]@polito.it

2 Massachusetts Institute of Technology, Cambridge, MA 02139, USA, LMainini@mit.edu is considered for the crashworthiness analysis, to assess the feasibility of powertrain configurations. Results show that, including crashworthiness analysis in the MDO formulation of the design problem, the identified optimal design differs from the outcome of a powertrain-only based optimization process. Particularly, benefits in terms of global solution feasibility and associated computational cost are achieved. The presented methodology allows to efficiently integrate powertrain analyses and crashworthiness constraints and it is suited for the early design of HEVs.

\section{Introduction}

Electrification of road vehicles is among the most effective solutions to comply with fuel economy targets and $\mathrm{CO} 2$ emission limits over the next few years (Bilgin et al. (2015)). As a matter of fact, powertrain electrification contributes to higher performance and more efficient road vehicles (Emadi (2011)). Example of specific contributions include (Kawaguchi et al. (2019)): (1) the mechanical to electrical energy conversion by means of regenerative braking; (2) automatically shutting off internal combustion engine (ICE) to reduce energy loss; (3) operating the ICE more efficiently.

ICE vehicles are to be redesigned into Hybrid Electric Vehicles (HEV) in order to satisfy pollution constraints and customers requirements. However, the presence of batteries and of additional power components (i.e. electric machines and inverters) as well as the increase in the total supplied power consistently affects the vehicle design. Particularly, an expansion of powertrain global envelope and a signficant increase of vehicle mass are observed. Consequently, structural design with respect to crashworthiness requirements has to be reconsidered.

In this study, we address the conversion of a conventional ICE vehicle into a HEV. The electrical components are commonly sized to minimize fuel consumption through a suited choice of the powertrain energy management strategy (Sil- 
vas et al. (2017)). The additional electrical components, such as the motor/generators MG1 and MG2 depicted in Figure 1, increase the total supplied power, resulting in an expansion of the demanded powertrain envelope. In addition, the electrical components and the batteries together contribute to significantly increase the overall mass of the vehicle, which in turn affects the response of the vehicle in case of impact. In impact events, the structure of the vehicle must absorb the kinetic energy through deformation of its structural components. In particular, the deformation of frontal crash boxes allows to prevent any intrusion in the cabin, guaranteeing passengers safety. Larger vehicle mass results in an increased kinetic energy to absorb in case of accidents. As a consequence, the structural framework devoted to the absorption of kinetic energy consistently expands its envelope in order to meet the safety requirements. However, for the constraints on vehicle widthtrack, the expansion of the structure framework results in conflicts with the need for lager powertrain envelope. The feasibility of the system is thus compromised and constitutes a major challenge for the designers.

The feasibility of the system is particularly crucial in early design stages. At this time, engineering systems are usually decoupled into subsystems according to their function. Each subsystem is then optimized within specific constraints. However, early design stages are characterized by uncertainties associated with limited information and lack of knowledge about system configuration. These uncertainty contributions particularly affect constraint functions and compromise the robustness and reliability of the results computed with the optimization process (Duddeck and Wehrle (2015)). The system feasibility is thus challenged. The electrification of a conventional ICE vehicle involves many decisions, such as those related to the design of motor/generators and of vehicular structural framework, which result in turn in uncertainty contributions. Given the conflicting physics of the illustrated problem, assuring the system feasibility from early design stages is of critical importance. In addition, the optimization process is usually time-consuming, requiring several runs to assess the optimal design. The counterposed expansions of powertrain systems and structural framework thus stimulate the formulation of a specific strategy which allows to optimize performances related to the different design disciplines, while achieving a globally feasible solution.

This paper proposes a Multidisciplinary Design Optimization (MDO) framework specifically developed for the preliminary design of the HEV powertrain which accounts for crashworthiness requirements. The Multidisciplinary Optimization framework, here a Multidisciplinary Feasible (MDF) architecture, allows to transfer information related to the different disciplines. In particular, the constraint on vehicle widthtrack can be accounted, while optimizing each discipline. MDO approaches have been largely adopted in literature to consider design environments with multiple disciplines (Agte et al. (2010)). Common MDO procedures for the design of complex systems decompose a large design problem into smaller discipline-related sub-problems (Guarneri and Wiecek (2016)). These can be subsequently solved by adopting dedicated algorithms and numerical tools. Recent examples can be found in literature that apply the MDO approach in the field of electric vehicles: Zhao et al. (2018) developed an MDO architecture for the chassis integrated system of electric wheel vehicles. A variablefidelity MDO architecture was considered by Wang et al. (2017) to interface the powertrain efficiency with the battery life-time maximization for an electric vehicle. MDO architectures can be divided into single level (or monolithic) and multiple level, according to the optimization task being considered all at once or hierarchically distributed into different levels (Yi et al. (2008)). Among single level MDO architectures, the multidisciplinary feasible approach finds widespread applications. The main advantage of MDF is that the system optimizer deals only with design variables (not including discipline responses), thus limiting the size of the design space. Nevertheless, the MDF might exhibit increased computational burden to execute the complete multidisciplinary analysis at each design iteration (Hulme and Bloebaum (2000)). Therefore, special steps may have to be taken when applying MDF to the specific MDO problem.

In this paper, HEV powertrain performances and crashworthiness capability of the structural framework are simultaneously optimized. A feasible design configuration for the hybridized vehicle, which is in turn the globally optimal solution, is thus obtained. In particular, powertrain performances are evaluated when crashworthiness criteria are satisfied, which drastically reduces computational time. Crash behavior of structures is analyzed through a multi-fidelity approach, in order to accurately establish the feasibility of powertrain configurations, while containing the computational cost.

The paper is organized as follows: the problem setup is firstly detailed with reference to the different disciplines here considered. Then, the vehicle models for powertrain components sizing and crashworthiness study are discussed. The developed MDO architecture for the considered design problem is thus presented. Finally, the electrification of a road vehicle is addressed with and without the proposed methodology: simulation results are illustrated and conclusions are given.

\section{Problem setup and background}

The electrification of road vehicles requires novel vehicle design solutions which consider both the sizing of power components and the expansion of the vehicular structural framework to meet safety requirements. Recent studies have addressed the problem of vehicles electrification, analyzing 


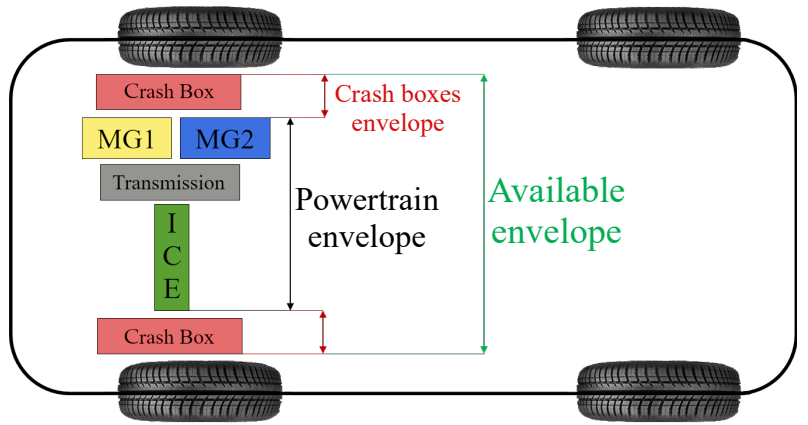

Figure 1: Powertrain and crash boxes envelopes in the transversal direction

both the powertrain and the structural framework. Dux et al. (2012) considered the electrification of a C-Segment car. They showed that the conversion from an ICE vehicle into a full electric vehicle affects the crashworthiness design of the structure. However, as the internal combustion engine is substituted with an electric drivetrain, the expansion of the structure framework does not result in conflicts with the envelope required by the electric powertrain. The design of mechanical components, aiming at increasing safety and reliability of battery packs, has been addressed in many works (Arora et al. (2016)). Other studies have proposed solutions for the crashworthiness design of front structures of electric vehicles (Lin et al. (2018)).

Thanks to the increasing computational power of modern calculators, transient nonlinear finite element analyses can be performed to assess crash behavior of structural components. Finite element analyss currently represents the most accurate and time-consuming response. These high-fidelity models are generally used in later design stages, when the structure is worth to be investigated with accurate numerical simulations (Duddeck and Wehrle (2015)). Structural optimization under crashworhtiness considerations requires several runs to assess the optimal design, which significantly increases the computational effort. In order to reduce the computational cost, which cannot be tackled only with faster computers, many approaches have been proposed over the years. Surrogate model based optimization is among the most used solutions. Surrogate models can be divided in two categories: mathematical surrogates and physical surrogates (Duddeck and Wehrle (2015)). According to the first approach, a mathematical surface is constructed as interpolation or regression of system responses. Example of this are reported by Avalle et al. (2002), where three different automotive structures subjected to impact are optimized through the response surface method. $\mathrm{Xu}$ (2014) proposed an extended surrogate modeling technique, embedding engineering knowledge. This resulted in both an increased accuracy and in a reduction of required samples. Other approaches consider local approximations of system responses in or- der to increase the accuracy of the surrogate model by reducing the design domain (Cadete et al. (2005), Hou et al. (2007)) and accounting for discontinuous responses with respect to the design domain (Boursier Niutta et al. (2018)). Physical surrogates are generally adopted in early development phases, due to the lack of knowledge. Physical surrogates can be based either on simplified version of accurate finite element models or on analytical and semi-analytical models. Wierzbicki and Abramowicz (1983) proposed one of the most used models of energy absorption mechanism of thin walled structures. Abramowicz and Jones (1984) developed theoretical predictions of axial progressive crushing, while Kecman (1982) studied theoretically the bending collapse of thin walled structures. Recent studies have used a combination of high- and low-fidelity models. By combining information coming from both models, it is possible to reduce computational effort without renouncing to accuracy (Jansson et al. (2003)).

In the electrification of ICE vehicles, the design of electrical components, i.e. the motor/generators and the transmission system, allows to manage power fluxes, optimizing performances. Many sizing methodologies for HEVs have been proposed in recent years. The sizing procedure and optimization algorithms relate to the analyzed HEV layout, particularly due to the choice of the powertrain energy management strategy (Silvas et al. (2017)). Considering series HEVs, Liu et al. (2007) presented a sizing methodology combining sequential quadratic programming with a genetic algorithm. The HEV optimization problem was approximated by a nonlinear convex problem by Murgovski et al. (2012), using heuristics for the ICE on/off operation and gear selection. The sizing procedure for a parallel HEV can be generally implemented using genetic algorithms (Madanipour et al. (2015)) or particle swarm optimization (Nüesch et al. (2012)). On the other hand, optimal component sizing of power-split HEVs exhibits increased complexity, especially in the case of multimode operation. Power-split architectures are the most successful and represent a large portion of the current population of HEV powertrains. They consist of one or multiple planetary gear (PG) sets, which are very compact and can realize a continuously variable transmission. The PG sets constitute the power split device (PSD), which is responsible for directing the power fluxes between the HEV powertrain components. Recently, a new trend considers the addition of clutches to a powersplit hybrid transmission in order to achieve different operating modes. Each specific case of vehicle operation (i.e. launching, accelerating, cruising at high speed, and regenerative braking) can be thus optimally suited. A common approach for the component sizing of these HEVs is represented by exhaustively searching in the considered design space (Zhuang et al. (2016), Anselma et al. (2019c)).

The problem of the conversion of standard ICE vehicles into 
HEV still has open questions. In particular, given the constraint on the vehicle widthtrack, the conflicting expansions of powertrain envelope and structural components have not been sufficiently addressed. This paper presents a multidisciplinary approach which allows to couple and account for information related to the different disciplines. The HEV powertrain performance and the crashworthiness capability of the vehicular structural framework are simultaneously optimized while accounting for the constraint on the vehicle widthtrack. The multidisciplinary approach leads to a feasible solution. A sequential scheme is adopted which evaluates crash performance first. The global optimizer defines at each iteration the powertrain configuration with its related envelope and mass increment. Different sizes are swept for the internal combustion engine, the electric motors and the planetary gear ratios through a direct search method. Then, crash behavior of structures is analyzed with a low-fidelity physical surrogate model, which allows for fast computations. Unfeasible candidates are then simulated through a high-fidelity finite element crash model in order to accurately establish their feasibility. When crashworthiness requirements are satisfied, powertrain performances are evaluated according to several drive cycles. Fuel consumption and acceleration performance are considered to assess powertrain performances and to design electrical components. The proposed approach thus leads to the optimal solution which guarantees the feasibility of all involved disciplines at once and simultaneously reduces the computational cost.

\section{Disciplinary level models}

In this section, each discipline model is presented and detailed. In regard to crashworthiness analysis, a multifidelity approach is adopted: low-fidelity and high-fidelity models are here described. HEV powertrain analysis is based on two different models for the evaluation of fuel consumption and acceleration performance, respectively.

\subsection{Crash-absorbing tube models}

In case of impact events, vehicle structure has to absorb the kinetic energy through deformation of its structural components. The deformation of frontal crash boxes allows to prevent any intrusion in the cabin, thus guaranteeing passengers safety. The electrification of the ICE vehicle involves larger vehicle mass. The kinetic energy to absorb in case of accidents thus increases as well. Consequently, structural components aimed to absorb energy (i.e. crash-absorbing tubes) have to be redesigned in order to meet safety requirements. Here, energy absorption capacity and peak force are considered as crashworthiness criteria.

In the present study, crash analyses are based on two different mechanical models: a low-fidelity physical surrogate model and a high-fidelity finite element model. While a surrogate formulation returns results at the lowest computational time, a finite element analysis is the most accurate. In particular, the low-fidelity physical model usually leads to conservative results in terms of absorbed energy and peak crushing force if compared to the finite element analysis (Liu and Day (2006), Kim et al. (1996)). For this reason, the low-fidelity model is firstly adopted to establish feasibility of each powertrain configuration. When crashworthiness criteria are not satisfied, analysis based on the high-fidelity model is performed, which improves accuracy of results. In addition, as time-consuming finite element analyses are performed only when required, the computational time is drastically reduced. Finally, performances of feasible powertrain candidates are evaluated. The overall MDO procedure will be detailed in section 4 .

\subsubsection{Low-fidelity mechanical model}

The low-fidelity crash model is a physical surrogate model, based on the analytical formulation derived by Wierzbicki and Abramowicz (1983), which describes the energy absorption mechanism of thin walled structures. The mean crushing force $F_{m}$ of a thin walled tube with rectangular cross-section is given by

$F_{m}=9.56 \cdot \sigma_{0} \cdot t^{\frac{5}{3}} \cdot\left(\frac{b+w}{2}\right)^{\frac{1}{3}}$

where $\sigma_{0}$ is the average flow stress, corresponding to $95 \%$ of the ultimate strength of crash box material, $b, w$ and $t$ the height, the width and the thickness of the crash box, respectively. The absorbed energy can be obtained by multiplying the mean force evaluated with equation 1 with a maximum allowable intrusion, here considered equal to 200 $\mathrm{mm}$. This value has been estimated assuming the presence of rigid bodies, such as the engine and the radiator, which do not allow any intrusion, as shown in Figure 2.

The peak force can be estimated from the buckling load of a rectangular cross-section thin walled tube, which is a function of the geometry, rather than the material strength. The buckling load of a thin plate compressed at the extremities is given by (Timoshenko and Gere (1963))

$\sigma_{c r}=k \frac{\pi^{2} E}{12\left(1-v^{2}\right)}\left(\frac{t}{w}\right)^{2}$

where $k$ is a coefficient depending on the ratio length over width of the plate, $E$ and $v$ the Young's modulus and the Poisson ratio of plate material, respectively, $t$ and $w$ the thickness and the width of the plate.

Equation 2 is the result of a equilibrium equation for the stability analysis of a compressed column. The stability analysis is based on the assumption of small displacements, which can no longer be considered for a collapsing column. In fact, after local buckling has occurred according to equation 2, the lateral deflections of the tube increase under increased 

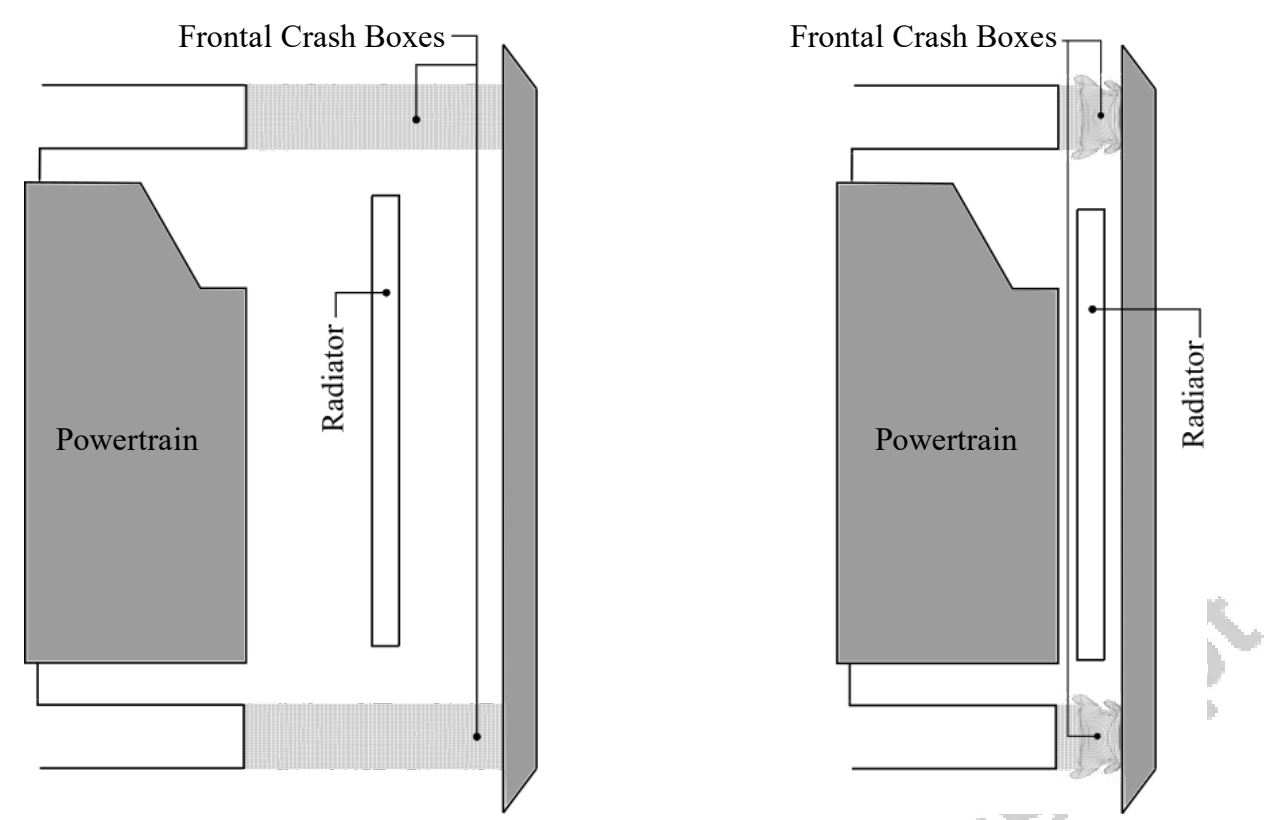

Figure 2: Schematic description of frontal crash boxes intrusion

loading. Large deflections leads to plasticity in the material. Therefore, the maximum load-carrying capacity of a column is controlled by a combination of geometry and material properties of the thin walled tube. The maximum strength is therefore (Mahmood and Paluszny (1981))

$\sigma_{\max }=\left(\frac{\sigma_{c r}}{\beta}\right)^{n} \sigma_{y}^{1-n}$

where $\sigma_{y}$ is the yield strength, $\beta$ is a parameter function of the material and of the ratio between $t$ and $w$ and $n$ is a geometry dependent parameter. For box-type columns, $n$ is equal to 0.43 (Mahmood and Paluszny (1981)). Substituting equation 2 in equation 3 and considering material properties of aluminum yields

$\sigma_{\max }=126\left(\frac{k}{\beta}\right)^{0.43}\left(\frac{t}{w}\right)^{0.86} \sigma_{y}^{0.57}$

Finally, the peak force is obtained by multiplying the resultant peak stress $\sigma_{\max }$ with the cross-sectional area of the crash tube

$F_{\max }=252 \cdot t^{1.86} \cdot w^{0.14}\left(\frac{k}{\beta}\right)^{0.43}\left(1+\frac{b}{w}\right) \sigma_{y}^{0.57}$

\subsubsection{High-fidelity mechanical model}

The high-fidelity mechanical model is based on a transient nonlinear finite-element analysis with explicit time integration. Numerical simulations have been performed using the commercial software LS-Dyna. The Belytschko-Tsay four node shell elements formulation with 6 degrees of freedom per node are used to model the structure. This formulation assumes three integration points through the thickness and one integration point in the element plane. The structure is discretized into about 30300 elements leading to overall $10^{5}$ degrees of freedom. The number of elements slightly varies according to the design of the considered crash box. The structure is impacted through a rigid wall with a non-zero coefficient and with an initial velocity of $13 \mathrm{~m} / \mathrm{s}$. The mass of the rigid wall is set according to the considered powertrain configuration. The corresponding vehicle mass allows to calculate the energy to absorb in case of impact, which is also the kinetic energy of the rigid wall. The aluminum alloy AW EN-6060 T6 is the material of the thin-walled structure, whose hardening curve in the plastic field is the same adopted by Wehrle (2015) in his models.

\subsection{HEV powertrain model}

\subsubsection{HEV powertrain model for fuel consumption evalua- tion}

The HEV multimode power-split powertrain retained in this paper comes from the industrial state-of-art and integrates two electric motor/generators (MGs) and an ICE (Pittel and Martin (2018)). The corresponding double PG lever diagram is reported in Figure 3. The HEV can operate in electric or hybrid mode according to the grounding clutch being engaged or disengaged.

In general, a vehicle model constituted by analytical equations is simulated in a backward quasi-static approach. Detailed modeling for the components of the considered HEV powertrain is presented as follows.

\section{Road load}

The vehicle speed profile corresponding to the driving 
mission under analysis can be taken as input for deriving the total horizontal resistance force acting on the vehicle body at each time step and is determined as

$F_{\text {road }}=R L_{A}+R L_{B} \cdot v+R L_{B} \cdot v^{2}$

where $F_{\text {road }}$ represents the total road load acting on the wheels, determined according to the actual vehicle speed $v$ and the empirical road load coefficients $\left(R L_{A}, R L_{B}\right.$ and $R L_{C}$ ) measured from vehicle coast down tests. The resistance torque $T_{\text {load }}$ requested to propel the vehicle satisfying the acceleration constraint is then determined as

$T_{\text {load }}=\frac{F_{\text {road }} \cdot r_{d y n}+\frac{I_{v} \cdot a}{r_{d y n}}}{\tau}$

where $r_{d y n}, I_{v}, a$ and $\tau$ are respectively the wheel rolling radius, the vehicle longitudinal inertia, the vehicle acceleration (evaluated from the value of vehicle speed in adjacent driving cycle points) and the transmission final drive ratio.

\section{Transmission}

In Figure 3, the MG2 operating speed $\omega_{M G 2}$ is cinematically constrained from the output speed $\omega_{\text {out }}$ while the MG1 speed $\omega_{M G 1}$ is a function of the ICE speed $\omega_{I C E}$ (which constitutes a control variable). The kinematic constraints can thus be summarized as

$\left[\begin{array}{l}\omega_{M G 1} \\ \omega_{M G 2}\end{array}\right]=\left[\begin{array}{cc}-r_{1} & r_{1}+1 \\ r_{2}+1 & 0\end{array}\right]\left[\begin{array}{c}\omega_{\text {out }} \\ \omega_{\text {ICE }}\end{array}\right]$

where $r_{1}$ and $r_{2}$ represent the transmission ratios between ring and sun of the PGs. When the ICE is grounded through the clutch in electric operation, $\omega_{I C E}$ is set to 0 . Assuming unitary efficiency for the transmission, the torque split between the two PGs can be determined arbitrarily satisfying the output energy demand.

\section{Power components}

Once the torque and speed values are determined for the MGs, the requested battery output power $P_{\text {batt }}$ can be evaluated as

$P_{b a t t}=\sum_{k=1}^{2} \omega_{M G k} \cdot T_{M G k} \cdot \eta_{M G k}^{-\operatorname{sign}\left(T_{M G k}\right)}$

where $\eta_{M G k}$ is the efficiency of each MG unit, evaluable by numerical efficiency maps including inverter efficiencies. The rate of battery State-of-Charge (SOC), SOC , can thus be calculated adopting an equivalent open circuit model

$S \dot{O} C=\frac{\sqrt{V_{O C}^{2}-4 \cdot R_{I N} \cdot P_{b a t t}}-V_{O C}}{2 \cdot R_{I N} \cdot Q_{b a t t}}$

where $V_{O C}, R_{I N}$ and $Q_{b a t t}$ are the output voltage, the internal resistance and the capacity of the battery, respectively. The fuel consumption can be evaluated as well from an ICE experimental furl flow map with torque and speed as independent variables.

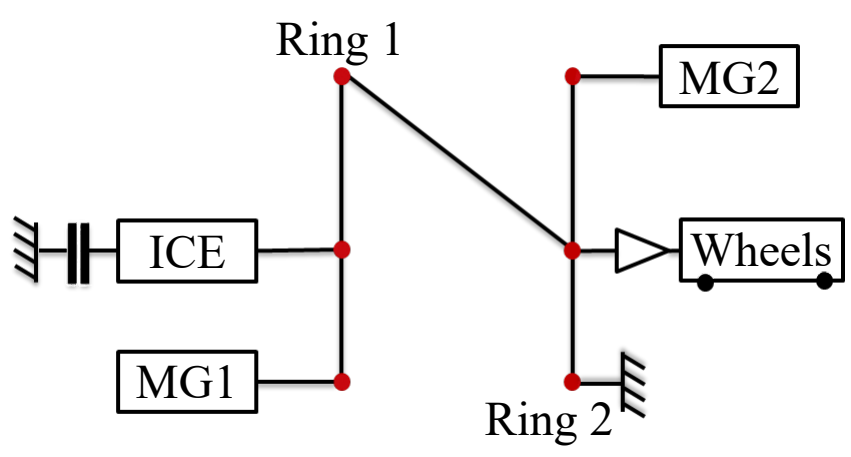

Figure 3: Lever diagram of the HEV powertrain under study

\subsubsection{HEV rapid control}

In order to assess the fuel economy capability of the analysed HEV candidate design, a proper control strategy needs implementation. In early vehicle design phases, the powertrain operation is optimized off-line. In other words, the trajectory of the vehicle speed is known a priori and it is determined by standard duty cycles. The mathematical formulation for the optimal control problem of an HEV can be stated as follows

$$
\begin{array}{ll} 
& \min _{\underline{x} \in \chi}\{f(\underline{x})\} \\
\text { where } & f=\int_{t_{0}}^{t_{\text {end }}} L\left(\omega_{I C E}, T_{I C E}, t\right) d t \\
& \underline{x}=\left[I C E M G 1 M G 2 r_{1} r_{2} r_{f d}\right]^{T} \\
\text { such that } & S O C\left(t_{0}\right)=S O C\left(t_{\text {end }}\right) \\
& \omega_{I C E_{\min }} \leq \omega_{I C E} \leq \omega_{I C E_{\max }} \\
& \omega_{M G 1_{\min }} \leq \omega_{M G 1} \leq \omega_{M G 1_{\max }} \\
& \omega_{M G 2_{\min }} \leq \omega_{M G 2} \leq \omega_{M G 2_{\max }} \\
& T_{I C E_{\min }} \leq T_{I C E} \leq T_{I C E_{\max }} \\
& T_{M G 1_{\min }} \leq T_{M G 1} \leq T_{M G 1_{\max }} \\
& T_{M G 2_{\min }} \leq T_{M G 2} \leq T_{M G 2_{\max }}
\end{array}
$$

where $L\left(\omega_{\text {ICE }}, T_{I C E}, t\right)$ represents the instantaneous rate of fuel consumption, which is evaluated by interpolation of the empirical lookup table considering ICE speed and torque as independent variables. The lookup table adopted in this work for the ICE fuel consumption is from Dabadie et al. (2017). Charge-sustaining (CS) criteria is defined by imposing equivalent battery SOC values at the beginning and the end of the considered time period. The battery $S O C$ rate $(S \dot{O} C)$ is function of the current $S O C$ value and the speed and torque values for the two MGs. The operation of the power components, defined by the corresponding actual values of speed (i.e. $\omega_{I C E}, \omega_{M G 1}$ and $\omega_{M G 2}$ ) and torque (i.e. $T_{I C E}, T_{M G 1}$ and $\left.T_{M G 2}\right)$, are restricted within their feasible regions. Particularly for the electric machines, both positive and negative torque values are allowed depending on their 
current operation (i.e. motor or generator respectively). Finally, limitations in the delivered or recharged battery power $P_{\text {batt }}$ are imposed accounting for its physical limits.

For the HEV retained in this paper, control variables are represented by the MG2 torque for the pure electric mode, while by the ICE speed and torque for the hybrid mode. Indeed, once these are known, speed and torque values for the remaining power components can be determined straightforwardly according to the lever diagram approach (Benford and Leising (1981)). In this regard, the most diffused approaches are the Pontryagin's minimum principle (PMP), the dynamic programming (DP) and the power-weighted efficiency-based analysis for rapid sizing (PEARS) (Wirasingha and Emadi (2011)). However, each of these presents some major drawbacks.

The PMP needs recursive calculation to tune the equivalence factor (Kim et al. (2012)), thus resulting inefficient in component sizing procedures. The DP approach examines the driving mission backwardly from its final time step back to the first one, evaluating the cost function for each discretized control value at each discretized state value (Anselma et al. (2019a)). A global optimal solution for the HEV fuel economy evaluation can be achieved in this way by operating an exhaustive search among all possible control actions at each time step of the driving mission (Lempert et al. (2018)). However, due to its operating principle, DP suffers from excessive computational cost (Liu and Peng (2008)). The PEARS algorithm was introduced to specifically deal with the component sizing problem of multimode power split HEVs (Zhang et al. (2015), Anselma et al. (2018)). Despite exhibiting results close to DP with consistently reduced computational effort, the PEARS algorithm may exhibit non-uniform proximity with the global optimum. This is observed particularly for HEVs with few operating modes, as the one here retained. In a recent work, Anselma et al. (2019b) analyzed the HEV illustrated in Figure 3 and proposed a novel control technique to overcome these limitations affecting DP and PEARS based approaches; the specific method is named slope-weighted energy-based rapid control analysis (SERCA) and allows to guarantee consistent proximity with the optimal fuel economy results provided by DP for the considered HEV, while remarkably reducing the related computational effort.

Following the SERCA procedure reported in Figure 4, discretized arrays for the control variables are firstly generated within the corresponding operating regions. By sweeping these arrays during Step 1, many possible solutions are generated for each time step of the analyzed drive cycle. These are labelled by the corresponding values of fuel and battery consumption according to the mathematical model introduced above. A cloud of possible solutions is created for each time step in this way. A related example for a time step can be observed in Figure 5, where the pure electric solu-

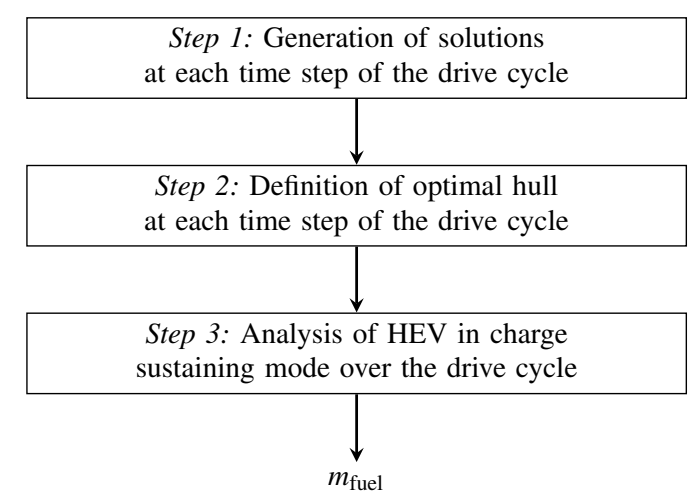

Figure 4: Scheme of the SERCA procedure

tions are distinguished by not consuming fuel.

The representation of Figure 5 can be interpreted as a sort of Pareto frontier for the considered time instant. Particularly, the solutions at the lower edge of the point cloud relate to the optimal ones, since they maximize the ratio between electrical energy generated and corresponding fuel energy consumed. The SERCA algorithm therefore considers these solutions for the eventual hybrid operation of the HEV in the drive cycle. As in Figure 5, the fuel consumption interval which comprehends all the possible solutions is firstly discretized. Then, a discrete optimal hull for each time point is obtained during Step 2 by considering the optimal solution for each element of the fuel consumption vector. Each element of the discrete hull is furthermore associated with the slope parameter, defined as the inclination of the line connecting two consecutive hull points.

The final phase of SERCA (i.e. Step 3) aims at analyzing the CS operation of the HEV in the drive cycle. As in PEARS, it is first assumed that the drive cycle is completed in pure electric operation. The total requested electrical energy is evaluated in this way as the sum of the energy values for the single time points. Then, an iterative process starts that aims at replacing pure electric with hybrid operation in the most convenient time points. These are identified based on the highest slope parameter. After an electric-to-hybrid replacement is performed in a particular time point, its corresponding slope parameter is updated considering the second element of the discretized hull. In general, the subsequent element of the discretized hull related to a particular time point is retained after this is selected for replacement. Meanwhile, the electrical energy to conclude the rest of the drive cycle in pure electric mode is updated. This recursive process is rapid and continues until CS operation is reached (i.e. the amount of cumulated electrical energy is 0). It has been demonstrated that, adopting this procedure, obtained fuel economy results are consistent with DP, while remarkably reducing the associated computational cost (Anselma et al. (2019b)). 


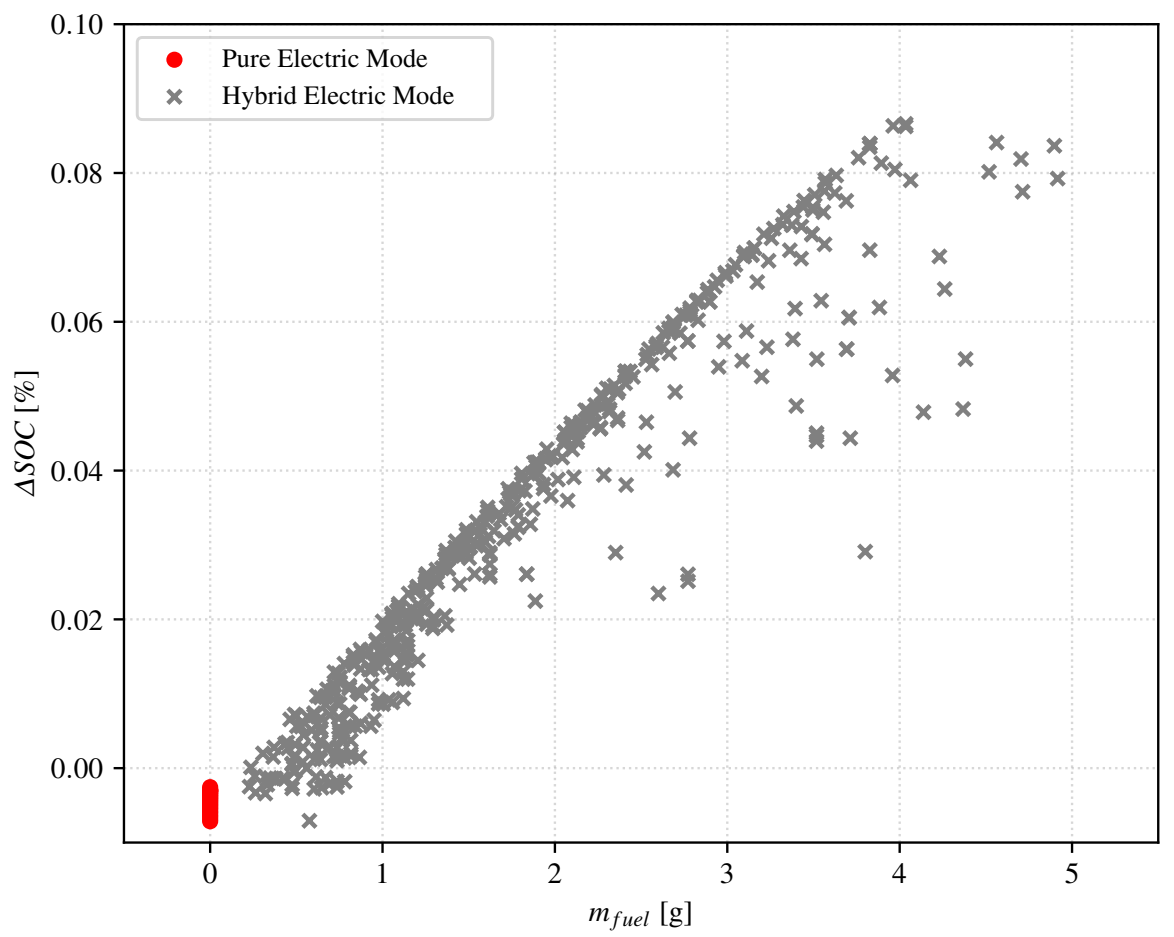

Figure 5: Example of possible solutions for a driving time step

\subsubsection{HEV powertrain model for acceleration evaluation}

In this paper, a MATLAB/Simulink@ model is employed to simulate each HEV candidate design during a $0-100 \mathrm{~km} / \mathrm{h}$ full throttle maneuver (Figure 6). As shown in the upperright zoomed view of the MG2 block, a lookup table is employed for MG2 which maps the maximum allowed deliverable torque as a function of the angular speed of the machine. In the PG1 subsystem, torques of ICE and MG1 are coordinated in such a way that the maximum possible torque is delivered at the ring gear output.

The total transmission output torque is then transferred at the front wheel blocks which evaluate the developed longitudinal tractive force. This information is elaborated by the vehicle body block, which calculates the actual value of vehicle speed and returns the amount of vertical load acting on the front tires. The tire blocks indeed incorporate a longitudinal behavior model based on the Pacejka's formulation with the tire longitudinal force depending on the corresponding vertical load (Bakker et al. (1989)). The vehicle speed value is set to $0 \mathrm{~km} / \mathrm{h}$ at the beginning of the simulation. Then, the simulation is carried until the vehicle speed reaches the value of $100 \mathrm{~km} / \mathrm{h}$. The recorded time value at which the simulation ends thus represents the time requested to complete the maneuver.

\section{Multidisciplinary feasible optimization of powertrain system and crash boxes}

A multidisciplinary approach is here adopted to simultaneously deal with component sizing and crash-absorbing tubes design. The optimal system is obtained by minimizing fuel consumption $\left(m_{f u e l}\right)$ while acceptable performances in terms of acceleration capability and crashworthiness are guaranteed. The optimization problem is formulated as follows

$$
\min _{\underline{x} \in \chi}\{f(\underline{x})\}
$$

where $f=m_{\text {fuel }}(\underline{x})$

$$
\underline{x}=\left[\begin{array}{lllll}
I C E & M G 1 & M G 2 & r_{1} & r_{2} \\
r_{f d}
\end{array}\right]^{T}
$$

such that $g_{1}=\frac{t_{0-100}}{t_{\text {allow }}}-1 \leq 0$

$$
g_{2}=\frac{2 \cdot w^{*}(\underline{x})+e_{H E V}(\underline{x})}{e_{\text {allow }}}-1 \leq 0
$$

The design variables vector $\underline{x}$ includes the operating maps of $I C E, M G 1$ and $M G 2$ (i.e. the internal combustion engine and electric motor/generators) and by $r_{1}, r_{2}$ and $r_{f d}$, which represent the trasmission ratios for the first planetary gear, second planetary gear and final drive, respectively. $g_{1}$ and $g_{2}$ denote the acceleration and envelope constraints, respectively. Particularly, the maximum acceptable 


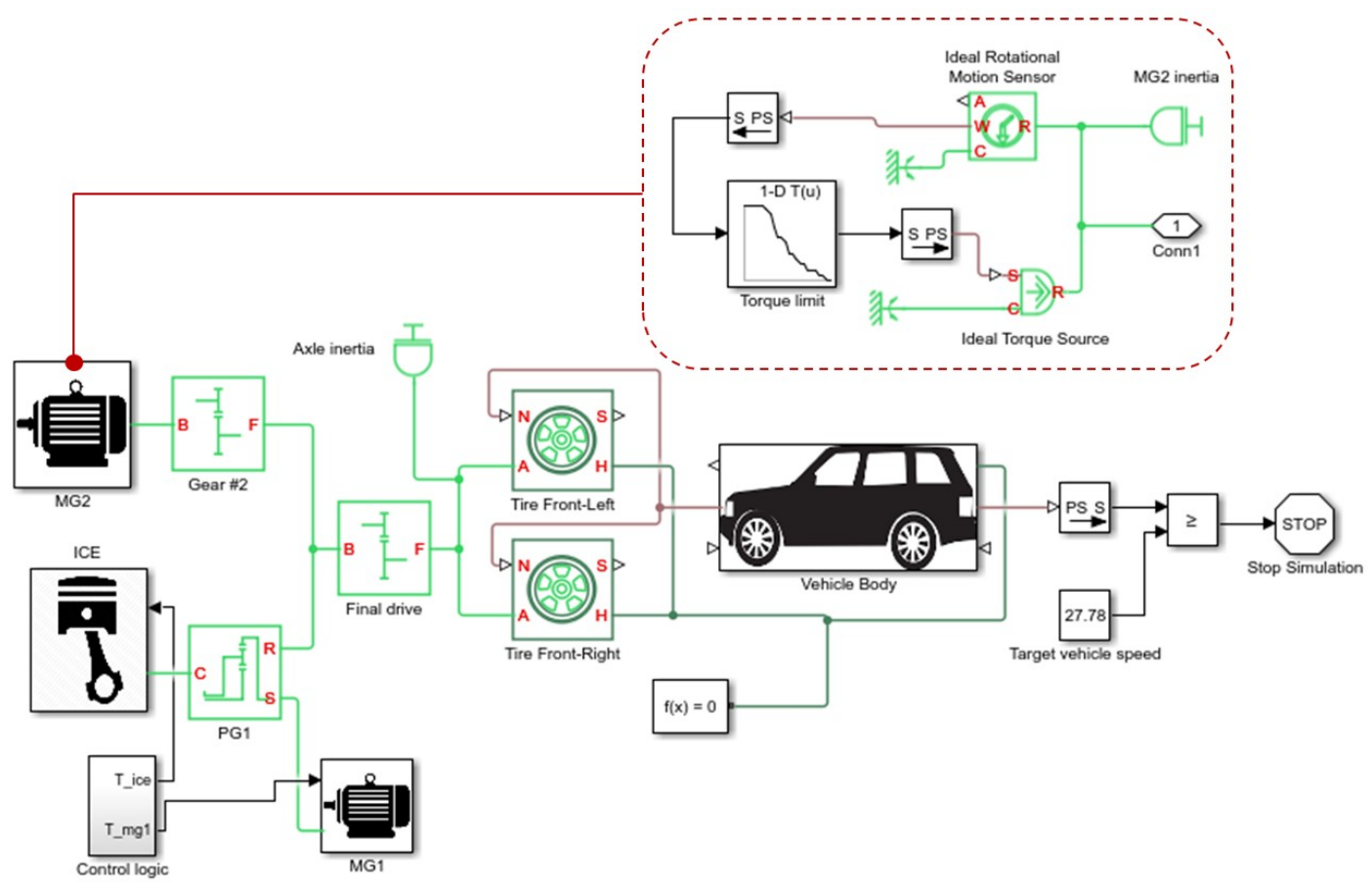

Figure 6: MATLAB/Simulink@ model for acceleration evaluation

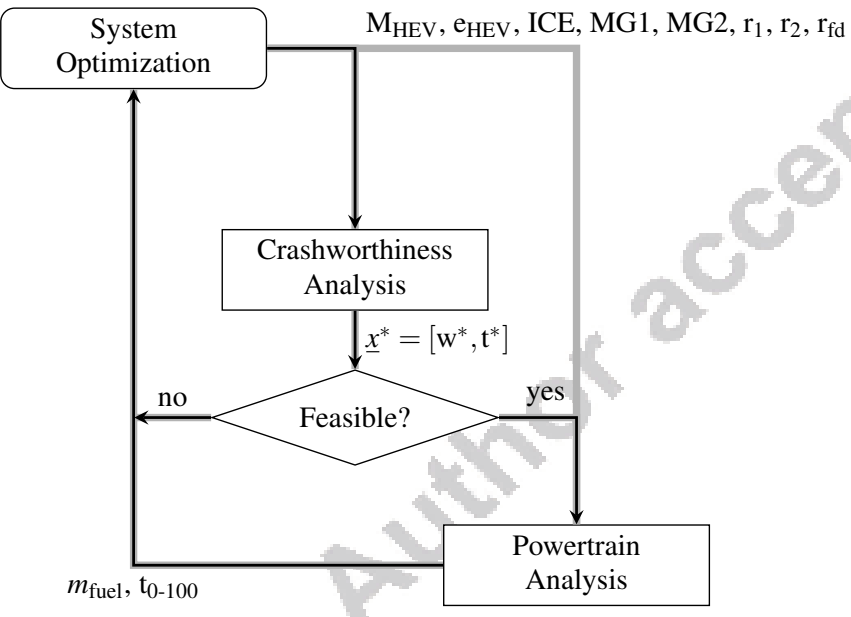

Figure 7: Multidisciplinary feasible optimization scheme

$0-100 \mathrm{~km} / \mathrm{h}$ acceleration time $\left(t_{\text {allow }}\right)$ is assumed equal to $10.0 \mathrm{~s}$.

In regard to safety requirements, the expansion of crash boxes due to the increased kinetic energy to absorb is limited by the total vehicle widthtrack. Here, the conflicting physics of the problem is addressed through a sequential scheme, shown in Figure 7. Particularly, we adopt a multidisciplinary feasible optimization approach, analyzing each discipline through a Gauss-Seidel scheme (Martins and Lambe (2013)). At each iteration, a powertrain configuration is defined. Mass of vehicle $\left(M_{H E V}\right)$ and envelope of powertrain
$\left(e_{H E V}\right)$ are specifically considered for the crashworthiness analysis. Then, optimal width $\left(w^{*}\right)$ and thickness $\left(t^{*}\right)$ of the crash boxes are calculated in order to meet safety requirements, in terms of energy absorption and maximum crash force. The sum of powertrain envelope $\left(e_{H E V}\right)$ and crashboxes width $\left(2 \cdot w^{*}\right)$ determines the feasibility of the considered configuration according to the total vehicle widthtrack $\left(e_{\text {allow }}\right)$. When both constraints $g_{1}$ and $g_{2}$ are satisfied, the powertrain analysis is performed. This sequential scheme is repeated for all powertrain configurations according to a direct search approach, leading to the optimum of the system, feasible for each discipline. A pseudocode of the proposed multidisciplinary optimization is presented in Algorithm 1.

4.1 Multi-fidelity crash-absorbing tube optimization

In the crashworthiness analysis, two design variables are considered: the width $w$ and the thickness $t$ of the crashabsorbing tube. The mass $m$ of the crash tube is firstly minimized, according to the low-fidelity surrogate model (Section 3.1.1). The structural optimization problem is formulated as follows 


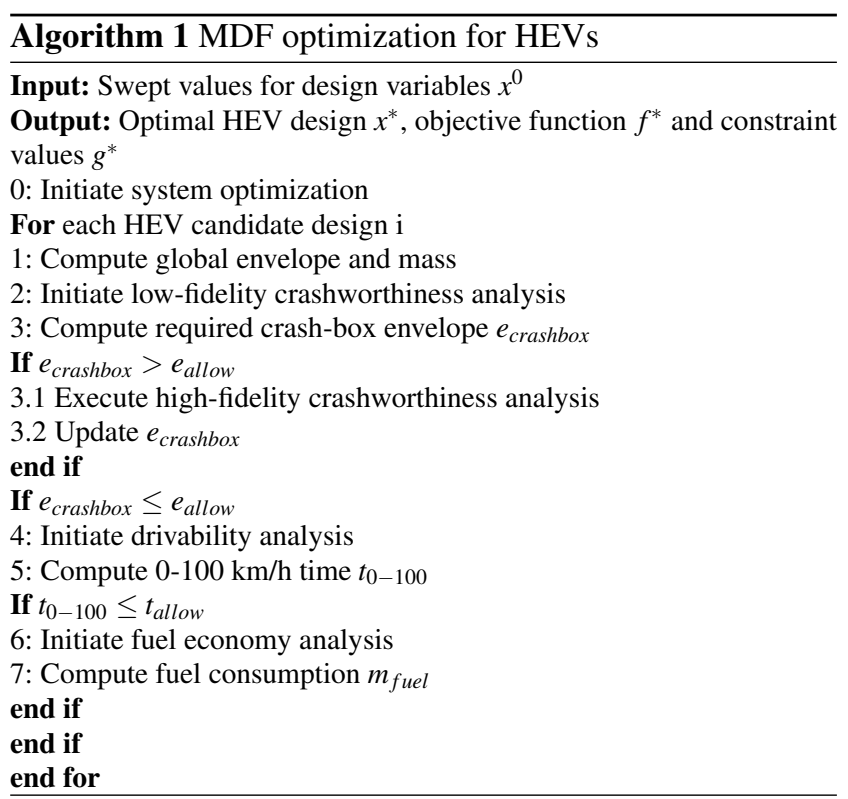

$$
\begin{array}{ll} 
& \min _{\underline{x} \in \chi}\{f(\underline{x})\} \\
\text { where } & f=m(\underline{x}) \\
& \underline{x}=[w t]^{T} \\
& 40.0 \leq w \leq 120.0 \mathrm{~mm} \\
& 1.5 \leq t \leq 1.75 \mathrm{~mm} .
\end{array}
$$$$
\text { such that } g_{1}=1-\frac{E_{a b s}(\underline{x})}{E_{a b s H E V}} \leq 0
$$$$
g_{2}=\frac{F_{\text {max }}(\underline{x})}{F_{\text {allow }}}-1 \leq 0
$$

According to the values assumed by the design variables $w$ and $t$ at each iteration of the optimization, the energy which can be absorbed through the retained configuration of the tube $E_{a b s}$ is estimated from equations 1 . The tubes have to absorb the kinetic energy of the impact through deformation. The energy to absorb $E_{a b s H E V}$ depends on the considered powertrain configuration, which defines the increment in the vehicle mass $\left(M_{H E V}\right)$. In addition, the peak force in the impact event $F_{\max }$ is limited. The numerical values for the state terms $E_{a b s H E V}$ and $F_{\text {allow }}$ are defined as follows. Given the energy absorption capacity of the original ICE vehicle $E_{a b s I C E}$ and the HEV mass $M_{H E V}$, the energy to absorb $E_{a b s H E V}$ is calculated as

$E_{a b s H E V}=\left(\frac{M_{H E V}}{M_{I C E}}\right) E_{a b s I C E}$

where $E_{a b s}$ and $M$ are the energy to absorb and the vehicle mass, respectively, and subscripts $H E V$ and $I C E$ refer to the hybrid and internal combustion engine vehicles, respectively. This study considers a structure made of aluminum alloy, with $\sigma_{0}=250 \mathrm{MPa}$. Crash box dimensions of original ICE vehicle have been assumed such that height, width and thickness are $110 \mathrm{~mm}, 65 \mathrm{~mm}$ and $1.5 \mathrm{~mm}$ respectively. Therefore, according to equation 1 and considering the maximum allowable intrusion of $200 \mathrm{~mm}$, the energy absorption capacity $E_{\text {abs ICE }}$ of each crash box is about $4000 \mathrm{~J}$. The reaction force is limited to the value $F_{\text {allow }}=100 \mathrm{kN}$.

The actual dimensions of crushing components are usually affected by manufacturing process. For instance, the extrusion of tubular geometries involves limitations on the thickness. Values reported in equation 13 are typical for crashabsorbing tubes. In regard to crash boxes height, this is assumed equal to $110 \mathrm{~mm}$, as for the original ICE vehicle.

Figure 8 shows the multi-fidelity scheme for crashworthiness analysis which benefits from the low-fidelity model (Section 3.1.1) in terms of computational cost and from the high-fidelity model (Section 3.1.2) in terms of accuracy. As finite element analysis is significantly time-consuming, the mass $m$ is firstly minimized according to the formulation 13 and the low-fidelity physical model is here considered to assess crashworthiness criteria. The first-order gradient-based algorithm COBYLA (Powell (1994)) has been utilized for the optimization. The information related to powertrain envelope, optimized width of crash boxes and total available envelope determines the feasibility of the retained powertrain candidate. When the feasibility of the powertrain configuration is not guaranteed, the high-fidelity model based analysis is performed. The use of the high-fidelity model at this stage allows to improve the accuracy of the result. In addition, as the time-consuming finite element analysis is performed only for discarded powertrain candidates, the computational time of the crashworthiness analysis is consistently contained. The transient nonlinear finite element model of the crash tube is simulated in LS-Dyna environment. The dimensions of the structural components are defined as follows: thickness value is assumed from the lowfidelity optimization $\left(t^{*}\right)$, while the tube width $w$ is set to the maximum allowable value $w_{\max }$,avail , determined from the powertrain envelope of the considered configuration and the total vehicle widthtrack. The feasibility of the powertrain configuration is thus established through the high-fidelity model, by comparing absorbed energy and reaction force to the corresponding state limit. When the crashworthiness criteria are not satisfied, the powertrain candidate is considered unfeasible and definitely discarded. Therefore, the high-fidelity model allows to assess the feasibility of the system with ameliorated accuracy, while the low-fidelity model is exploited to rapidly identify feasible configurations. The multi-fidelity scheme for the crashworthiness analysis thus limits the computational cost, while meeting acceptable accuracy of results. 


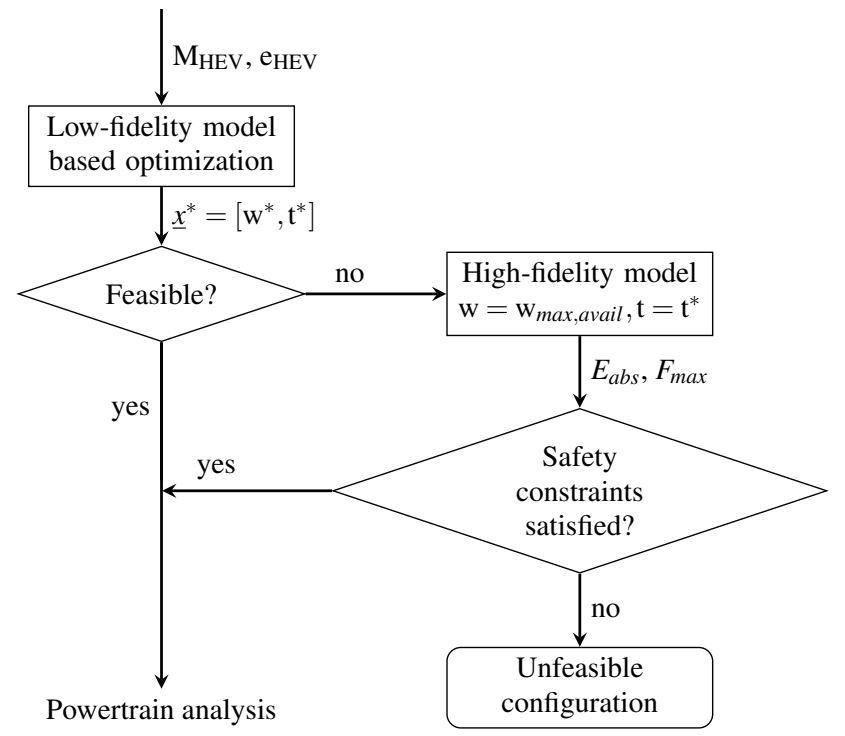

Figure 8: Multi-fidelity scheme for crashworthiness analysis

\subsection{Powertrain system analysis}

The HEV powertrain analysis considers two groups of design variables. The first relates to the power components (i.e. ICE and MGs) including their operational lookup tables and limit regions. The second involves the gear ratio parameters for the transmission. Once these are received as inputs, the drivability capability of the corresponding $\mathrm{HEV}$ is firstly assessed. This is performed by evaluating a $0-100 \mathrm{~km} / \mathrm{h}$ full throttle maneuver in MATLAB/Simulink ( environment. The analyzed HEV candidate design is furtherly considered or discarded depending on the obtained acceleration time being lower or greater than the imposed design constraint (10.0 $\mathrm{s}$ in this paper).

Then, in case the HEV candidate design is retained, fuel economy capability is assessed. Particularly, the HEV operation is simulated in different drive cycles. A near-optimal rapid off-line control strategy is employed here to rapidly estimate the fuel consumption of the HEV in the analyzed drive cycles. Consequently, the evaluated fuel consumption values for the drive cycles are weighted obtaining an averaged value representing the analyzed HEV candidate design. Finally, results are stored and the procedure is iterated considering a new candidate design.

\section{Results of the multidisciplinary optimization}

The electrification of a conventional ICE is carried out with and without the multidisciplinary feasible optimization, in order to point out the benefits of the proposed approach. Vehicle data considered in this paper are illustrated in Table 1. Characteristics of the baseline ICE vehicle are typical for several car segments. Therefore, the applicability of the proposed approach is not limited to specific vehicle design

\begin{tabular}{cccc}
\hline & Parameter & Value & Unit \\
\hline Baseline ICE & Mass & 1380 & $\mathrm{~kg}$ \\
vehicle data & Wheel radius & 0.3582 & $\mathrm{~m}$ \\
& ICE capacity & 2.0 & 1 \\
& ICE mass & 130 & $\mathrm{~kg}$ \\
Battery data & Capacity & 1000 & $\mathrm{~mm}$ \\
Optimization & ICE sizes (capacity) & $1.2 ; 1.4 ; 1.8$ & $\mathrm{kWh}$ \\
parameters & MG1 sizes (power) & $10 ; 20 ; 35 ; 45 ; 60$ & $\mathrm{~kW}$ \\
swept & MG2 sizes (power) & $45 ; 60 ; 75 ; 85 ; 95$ & $\mathrm{~kW}$ \\
& PG ratios (ring/sun) & $1: 1: 4$ & - \\
& $r_{f d}$ & $2.5: 1: 6.5$ & - \\
\hline
\end{tabular}

Table 1: Vehicle and powertrain data

cases.

For each candidate design, the HEV mass $\left(M_{H E V}\right)$ is calculated as the sum of the baseline ICE vehicle mass, the battery mass and the mass of the selected power components. Mass and dimensions of eletric motors MGs are linearly related to the corresponding power ratings reported in Table 1 as indicated by Finesso et al. (2018). Details concerning the retained MGs can be found in Anselma and Belingardi (2019). For the HEV, an additional constant mass of 400kg has been retained accounting for the battery pack, the related containment structure, the power electronics and the electrical wiring.

For the considered HEV, the total powertrain envelope $e_{H E V}$ can thus be obtained

$e_{H E V}=\left(e_{I C E}+e_{M G 2}+e_{P G 2}+e_{F D}\right) \cdot S F$

where $e_{I C E}, e_{M G 2}, e_{P G 2}$, and $e_{F D}$ are the envelope values for the ICE, the MG2, the PG2 and the final drive, respectively. $S F$ represents a safety factor accounting for minor envelope contributions (e.g. roller bearings, seals, accessories, clutches). Here we assume $S F=1.2$ MG1 and PG1 are not considered in the calculation of the powertrain envelope, as they are placed in parallel with respect to PG2 and MG2 (Pittel and Martin (2018)), as shown in Figure 1. Values for PG2 and final drive envelopes can be evaluated by following the standard preliminary gear design procedure based on Lewis' formulation (ISO-6336:2006 (E)).

The fuel economy capability is evaluated on four different drive cycles: (1) the urban dynamometer driving schedule (UDDS), (2) the highway fuel economy test (HWFET), (3) the new European driving cycle (NEDC) and (4) the worldwide harmonized light vehicle test procedure (WLTP). Drive cycles are analyzed separately. Then the overall estimated fuel consumption is represented by a weighted average of the single drive cycle contributions according to the following criterion: UDDS (27.5\%), HWFET (22.5\%), NEDC $(20 \%)$, WLTP $(30 \%)$. The analysis of multiple drive cycles enhances the significance of the obtained results. In this regard, thanks to its computational efficiency, the use of the SERCA technique is determinant to rapidly evaluate the fuel economy of candidate designs in various driving conditions. 
The electrification of the baseline ICE vehicle is firstly performed without considering any constraint for the envelopes. The results obtained through the optimization process are reported in Figure 9, where feasible and unfeasible designs are discriminated according to the acceleration performance requirement. Totally 308 acceptable designs are identified out of the 1154 analyzed designs in this way.

Subsequently, the optimization of powertrain components is performed within the multidisciplinary feasible framework here proposed. The results are displayed in Figures 10 and 11. Out of the 308 feasible candidates identified above, only 177 designs are found satisfying the crashworthiness requirements as well. As shown in Figure 10, the optimal design previously identified is no more acceptable due to incompatibility with the crashworthiness requirements. Therefore, a new optimal design is determined that satisfies all the involved disciplines at once.

Table 2 illustrates the results for both the analyses and compares these to the performance of the baseline ICE vehicle. The electrification leads to a significant increase of vehicle mass. In particular, in the optimal design of powertrain-only analysis, which do not account for crashworthiness requirements, an increment of $34.28 \%$ with respect to the baseline ICE is obtained. In the multidisciplinary approach, vehicle mass is $33.91 \%$ larger. However, the HEVs designs consistently ameliorate both fuel economy and acceleration performance of the baseline ICE.

In Table 3, crashworthiness analyses of optimal HEVs designs are compared. The envelope of the powertrain-only optimal design results equal to $834.2 \mathrm{~mm}$ and is $3.3 \%$ larger than the MDF design $(807.5 \mathrm{~mm})$. The resulting widths $\left(w_{\text {max }}\right.$ avail $)$ of crash-absorbing tubes are $82.9 \mathrm{~mm}$ for the powertrain-only optimal HEV and $96.25 \mathrm{~mm}$ for the MDF optimal HEV, respectively. The kinetic energy $E_{a b s H E V}$ is calculated from equation 14, with the baseline ICE vehicle mass $M_{I C E}$ equal to $1380 \mathrm{~kg}$. In particular, in the powertrainonly optimal design, $5640 \mathrm{~J}$ must be absorbed by each crash tube. Crashworthiness analysis based on the high-fidelity model reveals that this amount of energy cannot be absorbed with the considered dimensions of the crash boxes. In particular, the width of the crash components is not sufficient. Instead, the multidisciplinary approach leads to a feasible optimum, as shown in Table 3.

Figure 11 shows the feasibility of the design configurations, discerning between low-fidelity and high-fidelity based evaluations. As it can be observed, the high-fidelity analysis is mostly performed for candidates with best acceleration and fuel consumption results. This is due to the fact that these configurations make use of the most cumbersome motors and transmission systems, in order to be the most performing. This is the case of the MDF optimal HEV, whose feasibility is assessed through the high-fidelity finite element model, as shown in Figure 10. In particular, the multidisci- plinary feasible optimum satisfies crashworthiness requirements through the high-fidelity analysis, further validating its use.

Figures 12 and 13 compare low- and high-fidelity models in terms of absorbed energies and peak forces, respectively. The comparison considers the crash boxes corresponding to the powertrain-only-based optimal configuration (left-hand side), and to the MDF-based optimal configuration (righthand side). Figure 12 shows that the low-fidelity model underpredicts the value of absorbed energy in both the configurations. The absorbed energies calculated with the two models are also compared to the state term $E_{a b s H E V}$ of equation 14. As shown in the figure, in the powertrain-only-based optimal design both the high- and low-fidelity models inform that the configuration would not absorb the required energy of $5640 \mathrm{~J}$; in the case of the MDF-based optimal configuration, only the prediction provided by the high-fidelity model satisfies the requirement of $5610 \mathrm{~J}$. Figure 13 compares the peak forces of the two representations. Even in this case, we can observe that the low-fidelity model underpredicts the value of the peak force with respect to the high-fidelity counterpart. As shown in the figure, the peak forces of both the representations are lower than the state limit of $100 \mathrm{kN}$.

Finally, in regard to the computational time, the powertrain analysis of a single candidate design takes approximately $3 \cdot 10^{2} \mathrm{~s}$ on a desktop with Intel Core i7-8700 $(3.2 \mathrm{GHz})$ and 32 GB of RAM. The high-fidelity crashworthiness analysis of a single design candidate takes less than $4 \cdot 10^{2} \mathrm{~s}$ on the same computing platform. As a consequence, a powertrainonly based optimization process lasts about 1230 minutes (twenty hours) for the 308 candidates. On the other hand, results of the powertrain optimization within the multidisciplinary methodology have been obtained in 710 minutes (twelve hours) for the 177 candidates. This further demonstrates the efficiency of the proposed approach.

\section{Conclusion}

This paper has analyzed the electrification of a conventional ICE vehicle into a HEV. The presence of additional components, such as electric motor/generators and battery system consistently expands the powertrain envelope. Further, these electrical components result in larger vehicle mass, which affects crash behavior of the structural framework. In particular, structural elements which are devolved to the absorption of the vehicle kinetic energy in case of accidents demand for larger envelopes. However, for the constraint on the vehicle widthtrack, the expansion of the structural components results in conflict with the need for larger powertrain envelopes.

This work examined different sizes of power components for the electrified powertrain (namely, internal combustion engine, electric motors, transmission gear ratios, final drive ratio) for a specific vehicle body and chassis. A Multidisciplinary Feasible approach has been here considered for the 


\begin{tabular}{|c|c|c|c|c|}
\hline & Baseline ICE & Powertrain-only optimal & MDF optimal HEV & Unit \\
\hline ICE size & 2.0 & $\begin{array}{ll}\mathrm{HEV} & 1.4\end{array}$ & 1.4 & 1 \\
\hline MG1 power & - & 20 & 20 & $\mathrm{~kW}$ \\
\hline MG2 power & - & 95 & 85 & $\mathrm{~kW}$ \\
\hline$r_{1}$ & - & 2 & 3 & - \\
\hline$r_{2}$ & - & 1 & 1 & - \\
\hline$r_{f d}$ & - & 4 & 4 & - \\
\hline Mass & 1380 & 1853 & 1848 & $\mathrm{~kg}$ \\
\hline$t_{0-100}$ & 10.2 & 9.09 & 9.02 & $\mathrm{~s}$ \\
\hline Fuel consumption (averaged) & 849.2 & 609.9 & 630.3 & $\mathrm{~g}$ \\
\hline Fuel consumption (NEDC) & 514.8 & 405.3 & 424.3 & $\mathrm{~g}$ \\
\hline
\end{tabular}

Table 2: Electrification of the baseline ICE vehicle

\begin{tabular}{cccc}
\hline & Powertrain-only optimal HEV & MDF optimal HEV & Unit \\
\hline Mass & 1853 & 1848 & $\mathrm{~kg}$ \\
Powertrain axial envelope & 834.2 & 807.5 & $\mathrm{~mm}$ \\
Crash box width $w^{*}$ & 82.9 & 96.25 & $\mathrm{~mm}$ \\
Crash box thickness $t^{*}$ & 1.75 & 1.75 & $\mathrm{~mm}$ \\
14) & 5640 & 5610 & $\mathrm{~J}$ \\
Kinetic energy to absorb $E_{a b s H E V}$ (cf. Eq. & & & $\mathrm{J}$ \\
Absorbed energy (High-fidelity model) & 5321 & & $\mathrm{~J}$ \\
$E_{a b s}$ & & 96.540 & $\mathrm{kN}$ \\
Maximum Force (High-fidelity model) & 91.79 & & \\
$F_{\max }$ & &
\end{tabular}

Table 3: Comparison of crashworthiness analyses of HEV optimal designs

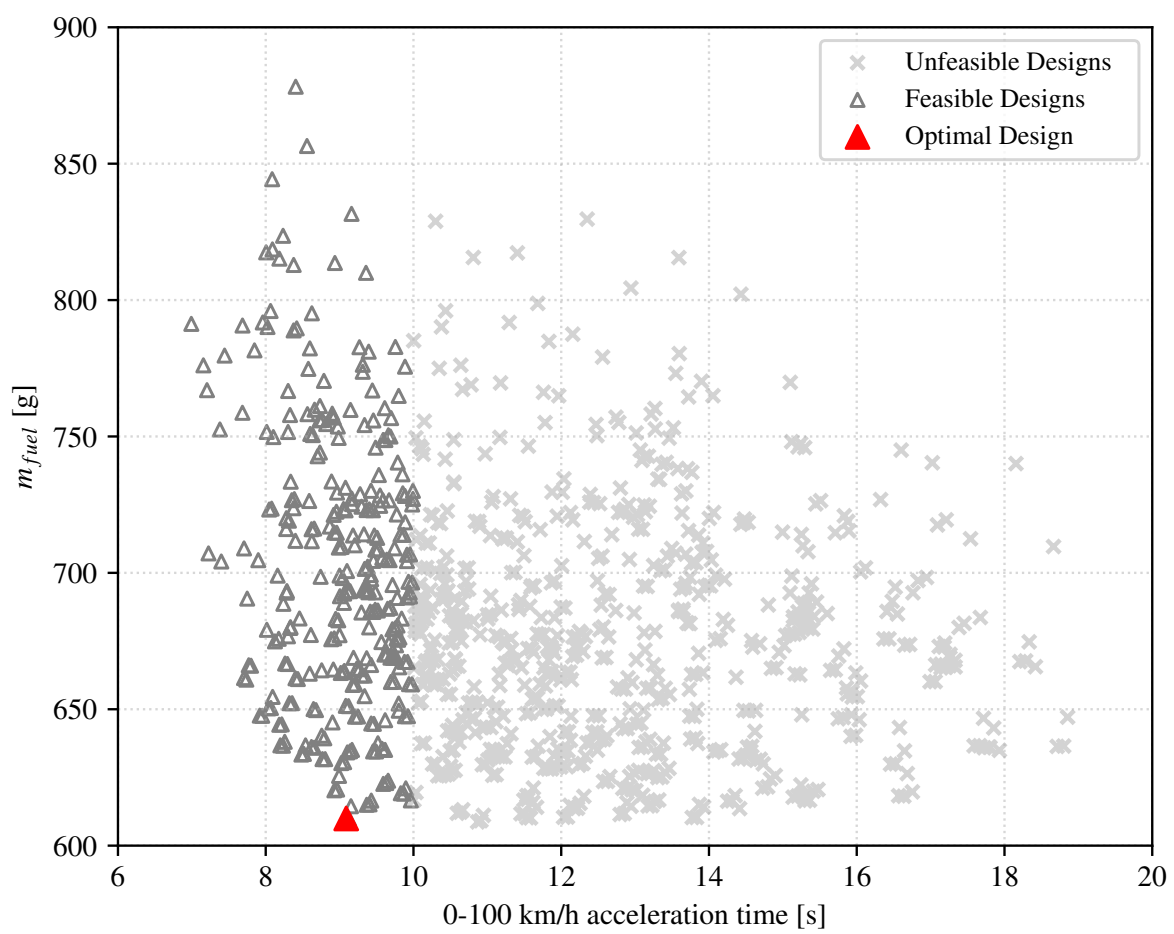

Figure 9: Feasibility with respect to acceleration performance 


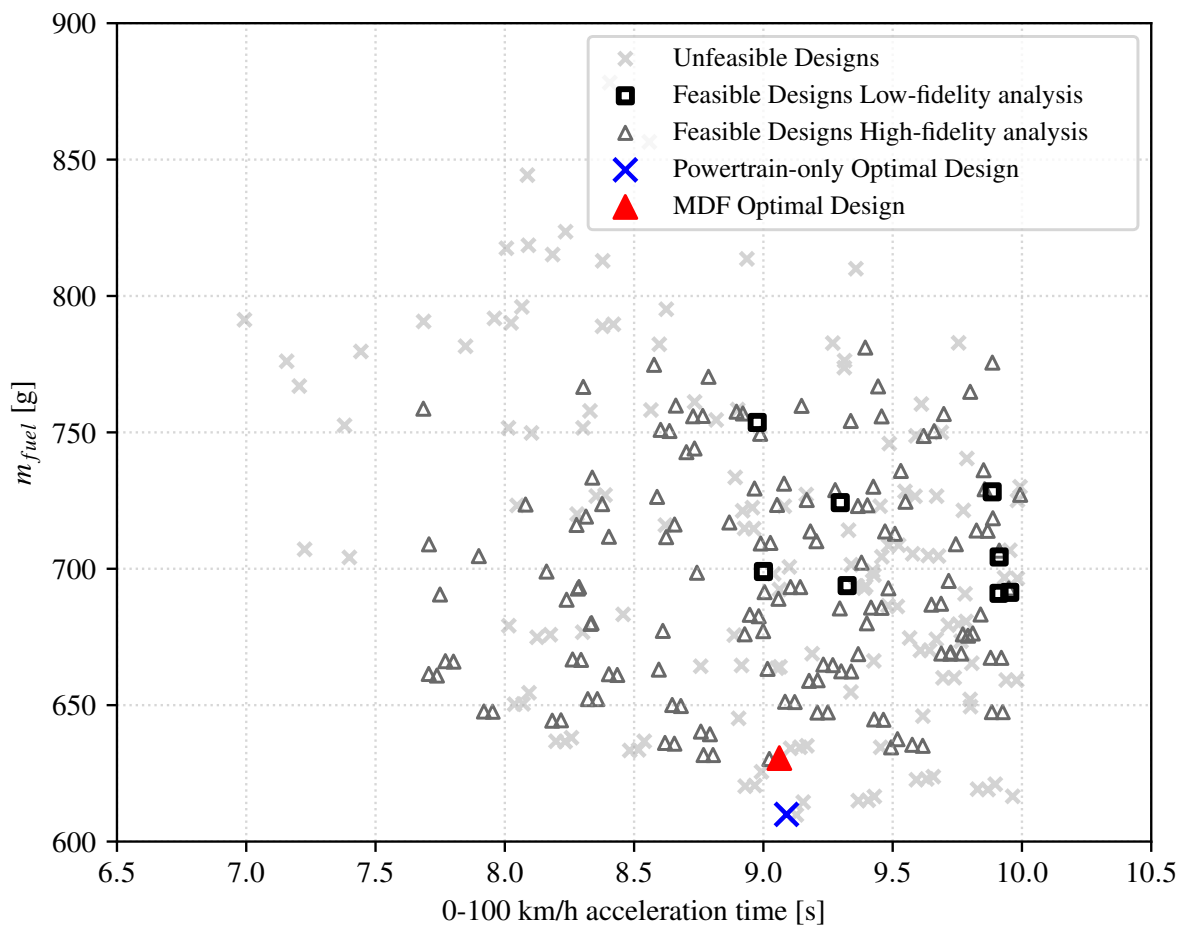

Figure 10: Optimization results according to the developed MDO approach

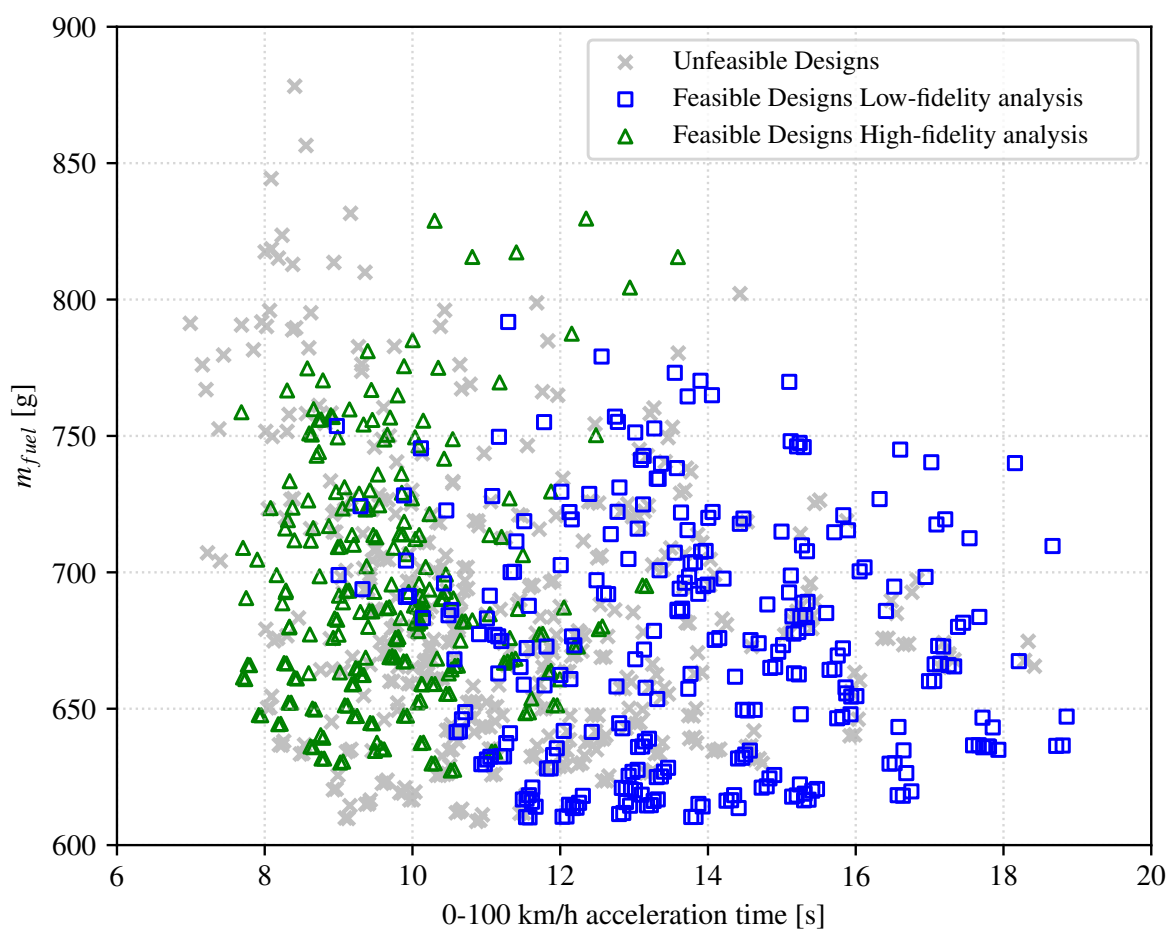

Figure 11: Feasibility assessment through crashworthiness analysis 


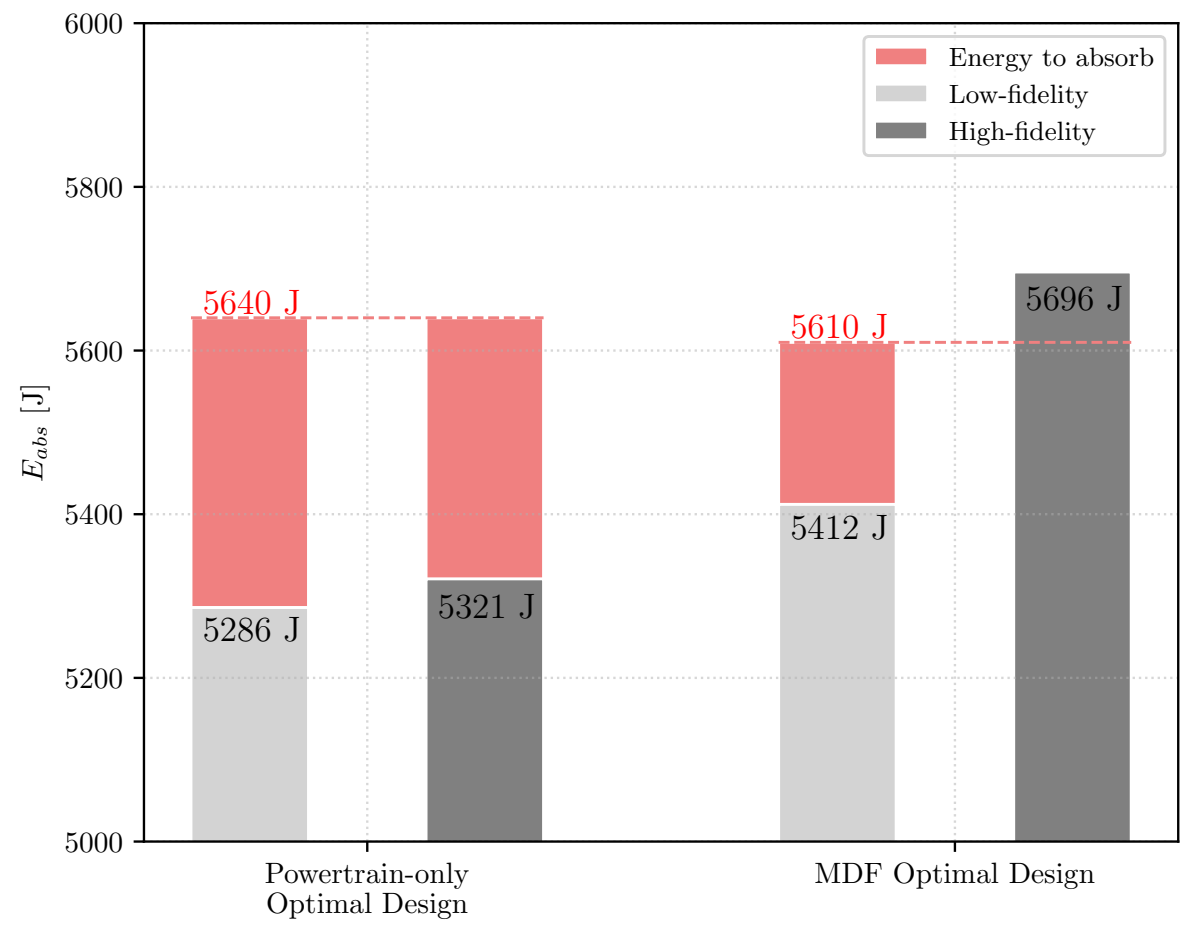

Figure 12: Comparison of the absorbed energy with the low- and high-fidelity models in the optimal design configurations

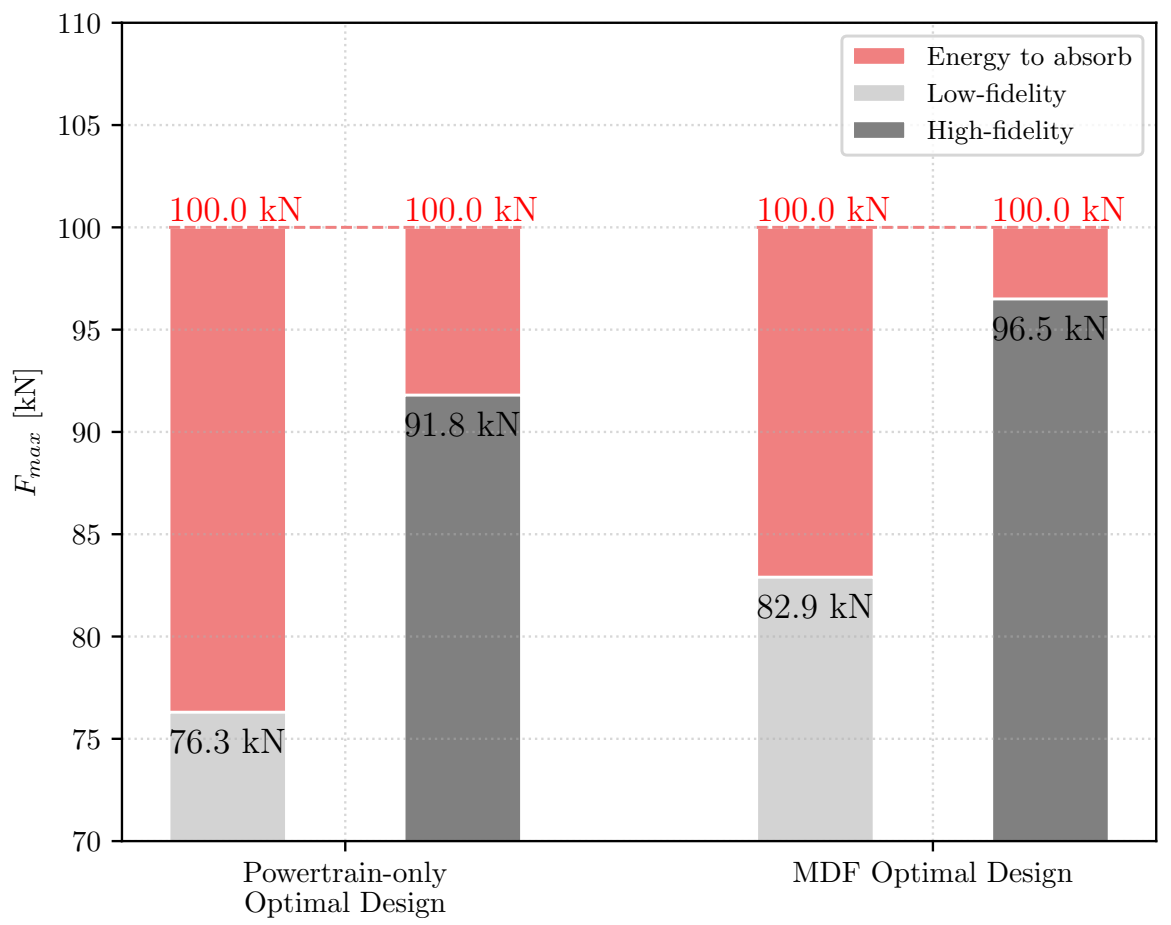

Figure 13: Comparison of the peak force with the low- and high-fidelity models in the optimal design configurations 
identification of the optimal configuration of the HEV powertrain. Crashworthiness analysis has been performed with a multi-fidelity approach, which involves a semi-analytical physical surrogate and a finite element model as low-fidelity and high-fidelity models, respectively. The multi-fidelity scheme for the crashworthiness analysis has allowed to contain the computational cost, while assuring satisfactory accuracy of results. The low-fidelity model has been firstly adopted to assess the feasibility of each powertrain candidate. When the feasibility was not guaranteed, the finite element model was simulated, thus improving the accuracy of the result. Feasibility of powertrain configurations has been thus assessed before powertrain analysis were performed. Restricting the pool of sizing options of the powertrain analysis using the crashworthiness analysis in the first place resulted convenient in this work. Given the comparable computational cost between the two disciplines, the amount of configuration options for the powertrain system is indeed considerably larger compared to the crashworthiness designs. This relates to the width of the gears limitedly affecting the overall powertrain envelope.

Results show that, including crashworthiness analysis in the MDO formulation of the design problem, the identified optimal design differs from the outcome of a powertrain-only based optimization process. For the retained vehicle and driving data, the optimal HEV layout obtained by means of a powertrain-only based procedure exhibits an averaged fuel consumption value of $609.9 \mathrm{~g}$ and a powertrain axial envelope of $834.2 \mathrm{~mm}$. However, the remaining envelope for the crash boxes in this case is demonstrated unsatisfactory to absorb the desired amount of kinetic energy during impact events. On the other hand, the proposed MDF approach for power split HEVs enables the identification of the optimal powertrain layout in terms of fuel economy while complying with the mentioned crashworthiness requirements. The MDF optimal HEV layout particularly reduces the powertrain envelope to $807.5 \mathrm{~mm}$. This is achieved at the expense of an averaged fuel consumption increased by $3.34 \%$ compared with the powertrain-only based optimum. Other than achieving benefits in terms of global solution feasibility, the illustrated design procedure enables narrowing the overall computational cost by $42.3 \%$. In general, the presented methodology allows to efficiently integrate powertrain analyses and crashworthiness constraints and it is suited for the early design of HEVs.

Related future work could consider additional crashworthiness analyses which account for the side crash requirements, the pole crash requirements and structures around the battery pack, as example. Moreover, further HEV powertrain architectures could be retained in the proposed MDF scheme. Finally, a multi-fidelity approach could be considered for the powertrain analysis as well.

\section{Conflict of Interest Statement}

On behalf of all authors, the corresponding author states that there is no conflict of interest.

\section{Acknowledgments}

This work was supported by the Doctoral School of Politecnico di Torino. Additional acknowledgments to the Visiting Professor Program of Politecnico di Torino for the support to Dr. Laura Mainini.

\section{Replication of results}

The method proposed in this paper can be implemented by following the pseudo code reported in Algorithm 1 and illustrated in Figure 7. The corresponding optimization problem is formulated in equations 11,12 and 13.

The disciplinary models are detailed in Section 3. The crashworthiness analysis is performed through equations 1 and 5, as illustrated in Figure 8, while equations 6-10 and illustration 6 refer to the hybrid powertrain evaluation.

\section{References}

W. Abramowicz and N. Jones. Dynamic axial crushing of square tubes. International Journal of Impact Engineering, 2(2):179-208, 1984.

J. Agte, O. de Weck, J. Sobieszczanski-Sobieski, P. Arendsen, A. Morris, and M. Spieck. Mdo: assessment and direction for advancement?an opinion of one international group. Structural and Multidisciplinary Optimization, 40 (17), 2010.

P. G. Anselma and G. Belingardi. Comparing battery electric vehicle powertrains through rapid component sizing. International Journal of Electric and Hybrid Vehicles, 11 (1):36-58, 2019.

P. G. Anselma, Y. Huo, E. Amin, J. Roeleveld, A. Emadi, and G. Belingardi. Mode-shifting minimization in a power management strategy for rapid component sizing of multimode power split hybrid vehicles. In SAE Technical Paper, 2018.

P. G. Anselma, G. Belingardi, A. Falai, C. Maino, F. Miretti, D. Misul, and E. Spessa. Comparing parallel hybrid electric vehicle powertrains for real-world driving. In 2019 AEIT International Conference of Electrical and Electronic Technologies for Automotive (AEIT AUTOMOTIVE), 2019a.

P. G. Anselma, Y. Huo, J. Roeleveld, G. Belingardi, and A. Emadi. Slope-weighted energy-based rapid control analysis for hybrid electric vehicles. IEEE Transactions on Vehicular Technology, 68(5):4458-4466, $2019 \mathrm{~b}$.

P. G. Anselma, Y. Huo, J. Roeleveld, A. Emadi, and G. Belingardi. Rapid optimal design of a multimode power split hybrid electric vehicle transmission. In Proceedings of the Institution of Mechanical Engineers, Part D: Journal of Automobile Engineering, 2019c.

S. Arora, W. Shen, and A. Kapoor. Review of mechanical design and strategic placement technique of a robust bat- 
tery pack for electric vehicles. Renewable and Sustainable Energy Reviews, 60:1319-1331, 2016.

M. Avalle, G. Chiandussi, and G. Belingardi. Design optimization by response surface methodology: application to crashworthiness design of vehicle structures. Structural and Multidisciplinary Optimization, 24(4):325-332, 2002.

E. Bakker, H. B. Pacejka, and L. Lidner. A new tire model with an application in vehicle dynamics studies. In $S A E$ Technical Paper, 1989.

H. Benford and M. Leising. The lever analogy: A new tool in transmission analysis. In SAE Technical Paper, 1981.

B. Bilgin, P. Magne, P. Malysz, Y. Yang, V. Pantelic, M. Preindl, A. Korobkine, W. Jiang, M. Lawford, and A. Emadi. Making the case for electrified transportation. IEEE Transactions on Transportation Electrification, 1 (1):4-17, 2015.

C. Boursier Niutta, E. J. Wehrle, F. Duddeck, and G. Belingardi. Surrogate modeling in design optimization of structures with discontinuous responses - a new approach for ill-posed problems in crashworthiness design. Structural and Multidisciplinary Optimization, 57(5):18571869, 2018.

Rui N. Cadete, Joao P. Dias, and Manuel S. Pereira. Optimization in vehicle crashworthiness design using surrogate models. In 6th World Congress of Structural and Multidisciplinary Optimization, 2005.

J. Dabadie, A. Sciarretta, G. Font, and F. Le Berr. Automatic generation of online optimal energy management strategies for hybrid powertrain simulation. In SAE Technical Paper, 2017.

F. Duddeck and E. J. Wehrle. Recent advances on surrogate modeling for robustness assessment of structures with respect to crashworthiness requirements. In 10th European LS-DYNA Conference, 2015.

E. Dux, B. Hartmann, S. Faßbender, P. Urban, L. Eckstein, and M. Bröckerhoff. Investigation of the trade-off between lightweight and battery cost for an aluminiumintensive electric vehicle, 2012.

A. Emadi. Transportation 2.0. IEEE Power and Energy Magazine, 9(4):18-29, 2011.

R. Finesso, D. Misul, E. Spessa, and M. Venditti. Optimal design of power-split hevs based on total cost of ownership and co2 emission minimization. Energies, 11(7), 2018.

P. Guarneri and M. Wiecek. Pareto-based negotiation in distributed multidisciplinary design. Structural and Multidisciplinary Optimization, 53(4):657-671, 2016.

S. Hou, Q. Li, S. Long, X. Yang, and W. Li. Design optimization of regular hexagonal thin-walled columns with crashworthiness criteria. Finite Elements in Analysis and Design, 43(6-7):555-565, 2007.
K. Hulme and C. Bloebaum. A simulation-based comparison of multidisciplinary design optimization solution strategies using cascade. Structural and Multidisciplinary Optimization, 19(1):17-35, 2000.

ISO-6336:2006(E). Calculation of load capacity of spur and helical gears. Standard, International Organization for Standardization, Geneva, CH, 2006.

T. Jansson, L. Nilsson, and M. Redhe. Using surrogate models and response surfaces in structural optimization - with application to crashworthiness design and sheet metal forming. Structural and Multidisciplinary Optimization, 25(2):129-140, 2003.

B. Kawaguchi, K. Umemoto, S. Misawa, S. Hirooka, and T. Kawai. Ice vehicle challenge toward zero emissions: future technology harmonization in electrified powertrain system. In SAE Technical Paper, 2019.

D. Kecman. Bending collapse of rectangular and square section tubes. International Journal of Mechanical Sciences, 25(9-10):623-636, 1982.

H. S. Kim, S. Y. Kangu, I. H. Lee, S. H. Park, and D. C. Han. Vehicle frontal crashworthiness analysis by simplified structure modeling using nonlinear spring and beam elements. International Journal of Crashworthiness, 2(1): 107-118, 1996.

N. Kim, S. W. Cha, and H. Peng. Optimal equivalent fuel consumption for hybrid electric vehicles. IEEE Transactions on Control Systems Technology, 20(3):817-825, 2012.

J. Lempert, B. Vadala, K. Arshad-Aliy, J. Roeleveld, and A. Emadi. Practical considerations for the implementation of dynamic programming for hev powertrains. In IEEE Transportation Electrification Conference and Expo, 2018.

C. Lin, F. Gao, and Y. Bai. Multiobjective reliabilitybased design optimisation for front structure of an electric vehicle using hybrid metamodel accuracy improvement strategy-based probabilistic sufficiency factor method. International Journal of Crashworthiness, 23(3):290-301, 2018.

J. Liu and H. Peng. Modeling and control of a power-split hybrid vehicle. IEEE Transactions on Control Systems Technology, 16(6):1242-1251, 2008.

X. Liu, Y. Wu, and J. Duan. Optimal sizing of a series hybrid electric vehicle using a hybrid genetic algorithm. In IEEE International Conference on Automation and Logistics, 2007.

Y. C. Liu and M. L. Day. Simplified modelling of thinwalled box section beam. International Journal of Crashworthiness, 11(3):263-272, 2006.

V. Madanipour, M. Montazeri-Gh, and M. Mahmoodi-k. Optimization of the component sizing for a plug-in hybrid electric vehicle using a genetic algorithm. In Proceedings of the Institution of Mechanical Engineers, Part 
D: Journal of Automobile Engineering, 2015.

H. F. Mahmood and A. Paluszny. Design of thin walled columns for crash energy management - their strength and their mode of collapse. In SAE Technical Paper, 1981.

J. R. R. A. Martins and A. B. Lambe. Multidisciplinary design optimization: A survey of architectures. AIAA Journal, 51(9):2049-2075, 2013.

N. Murgovski, L. Johannesson, J. Sjoberg, and B. Egardt. Component sizing of a plug-in hybrid electric powertrain via convex optimization. Mechatronics, 22(1):106-120, 2012.

T. Nüesch, T. Ott, S. Ebbesen, and L. Guzzella. Cost and fuel-optimal selection of hev topologies using particle swarm optimization and dynamic programming. In American Control Conference (ACC), 2012.

M. Pittel and D. Martin. eflite dedicated hybrid transmission for chrysler pacifica. In SAE Technical Paper, 2018.

M. J. D. Powell. A direct search optimization method that models the objective and constraint function by linear interpolation. In 6th Workshop on Optimization and $\mathrm{Nu}$ merical Analysis, 1994. Reprinted in Gomez, S. and Hennart, J.-P. (1994) Advances in Optimization and Numerical Analysis.

E. Silvas, T. Hofman, N. Murgovski, L. F. P. Etman, and M. Steinbuch. Review of optimization strategies for system-level design in hybrid electric vehicles. IEEE Transactions on Vehicular Technology, 6(1):57-70, 2017.

S. P. Timoshenko and J. M. Gere. Theory of elastic stability. McGraw-Hill, second edition, 1963.

X. Wang, M. Li, Y. Liu, W. Sun, X. Song, and J. Zhang. Surrogate based multidisciplinary design optimization of lithium-ion battery thermal management system in electric vehicles. Structural and Multidisciplinary Optimization, 56(6):1555-1570, 2017.

E. J. Wehrle. Design optimization of lightweight space frame structures considering crashworthiness and parameter uncertainty. Dr.-ing. diss., Lehrstuhl für Leichtbau, Technische Universität München, 2015.

T. Wierzbicki and A. Abramowicz. On the crushing mechanics of thin-walled structures. Journal of Applied Mechanics, 50:727-734, 1983.

S. G. Wirasingha and A. Emadi. Classification and review of control strategies for plug-in hybrid electric vehicles. IEEE Transactions on Vehicular Technology, 60(1):111122,2011

Q. Xu. Extended surrogate modeling techniques for large scale structural design optimization. Dr.-ing. diss., Lehrstuhl für Leichtbau, Technische Universität München, 2014.

S. I. Yi, J. K. Shin, and G. J. Park. Comparison of mdo methods with mathematical examples. Structural and Multidisciplinary Optimization, 35(5):391-402, 2008.
X. Zhang, H. Peng, and J. Sun. A near-optimal power management strategy for rapid component sizing of multimode power split hybrid vehicles. IEEE Transactions on Control Systems Technology, 23(2):609-618, 2015.

W. Zhao, Z. Yang, and C. Wang. Multidisciplinary hybrid hierarchical collaborative optimization of electric wheel vehicle chassis integrated system based on driver's feel. Structural and Multidisciplinary Optimization, 57:11291147, 2018.

W. Zhuang, X. Zhang, H. Peng, and L. Wang. Rapid configuration design of multiple-planetary-gear power-split hybrid powertrain via mode combination. IEEE/ASME Transactions on Mechatronics, 21(6):2924-2934, 2016. 